\title{
FACTORIZATION OF BIVARIATE SPARSE POLYNOMIALS
}

\author{
FRANCESCO AMOROSO AND MARTÍN SOMBRA
}

\begin{abstract}
We prove a function field analogue of a conjecture of Schinzel on the factorization of univariate polynomials over the rationals. We derive from it a finiteness theorem for the irreducible factorizations of the bivariate Laurent polynomials in families with fixed set of complex coefficients and varying exponents. Roughly speaking, this result shows that the truly bivariate irreducible factors of these sparse Laurent polynomials, are also sparse.
\end{abstract}

\section{INTRODUCTION}

A polynomial is said to be sparse (or lacunary) if it has few terms compared with its degree. The factorization problem for sparse polynomials can be vaguely stated as the question of whether the irreducible factors of a sparse polynomial are also sparse, apart from obvious exceptions. Aspects of this problem have been studied in various settings and for different formalizations of the notion of sparsenness, see for instance [Len99, Sch00, KK06, AKS07, FGS08, Gre16, ASZ17]. Several of these studies were based on tools from Diophantine geometry like lower bounds for the height of points and subvarieties, and unlikely intersections of subvarieties and subgroups of a torus.

In this text, we consider families of bivariate Laurent polynomials given as the pullback of a fixed regular function on a torus by a varying 2-parameter monomial map. Precisely, let $t, z$ be variables, $\boldsymbol{x}=\left(x_{1}, \ldots, x_{n}\right)$ a set of other $n$ variables, and

$$
F \in \mathbb{C}\left[\boldsymbol{x}^{ \pm 1}, z^{ \pm 1}\right]=\mathbb{C}\left[x_{1}^{ \pm 1}, \ldots, x_{n}^{ \pm 1}, z^{ \pm 1}\right]
$$

a Laurent polynomial. For each vector $\boldsymbol{a}=\left(a_{1}, \ldots, a_{n}\right) \in \mathbb{Z}^{n}$, we consider the bivariate Laurent polynomial given as the pullback of $F$ by the monomial map $(t, z) \mapsto\left(t^{a}, z\right)=$ $\left(t^{a_{1}}, \ldots, t^{a_{n}}, z\right)$, that is

$$
F_{\boldsymbol{a}}=F\left(t^{\boldsymbol{a}}, z\right) \in \mathbb{C}\left[t^{ \pm 1}, z^{ \pm 1}\right] .
$$

The number of coefficients of each $F_{\boldsymbol{a}}$ is bounded by those of $F$, and so these Laurent polynomials can be considered as sparse when $F$ is fixed and $\boldsymbol{a}$ is large.

A first question concerns the irreducibility of $F$. It has been addressed in [Zan10], as we next describe. Let us assume $F$ irreducible. Under which assumptions $F_{\boldsymbol{a}}$ stays irreducible for a generic $\boldsymbol{a}$ ? Let us consider the following example. The Laurent polynomial $F=z^{2}-x_{1} x_{2}^{2} \in \mathbb{C}\left[x_{1}^{ \pm 1}, x_{2}^{ \pm 1}, z^{ \pm 1}\right]$. is irreducible. However given $\left(a_{1}, a_{2}\right) \in$ $\mathbb{Z}^{2} \backslash\{(0,0)\}$ with $a_{1}$ even, the Laurent polynomial $F_{\boldsymbol{a}}$ is reducible. This show that the sole assumption that $F$ is irreducible is not enough to get an irreducibility statement for a generic specialization.

Zannier's result can be stated as follows. For $\boldsymbol{a}, \boldsymbol{b} \in \mathbb{Z}^{n}$, we denote by $\langle\boldsymbol{a}, \boldsymbol{b}\rangle=$ $\sum_{i=1}^{n} a_{i} b_{i}$ their scalar product.

Date: $12 / 9 / 2018$.

2010 Mathematics Subject Classification. Primary 13P05; Secondary 12 Y05.

Amoroso was partially supported by the CNRS research project PICS 6381 "Diophantine geometry and computer algebra". Sombra was partially supported by the MINECO research project MTM201565361-P. 
Theorem 1.1 ([Zan10, Theorem 3]). Let $F \in \mathbb{C}\left[\boldsymbol{x}^{ \pm 1}, z^{ \pm 1}\right] \backslash \mathbb{C}\left[\boldsymbol{x}^{ \pm 1}\right]$ be an irreducible Laurent polynomial, that is monic in $z$ and such that $F\left(x_{1}^{d}, \ldots, x_{n}^{d}, z\right)$ is irreducible for $d=\operatorname{deg}_{z}(F)$. There is a finite subset $\Sigma \subset \mathbb{Z}^{n} \backslash\{\mathbf{0}\}$ such that, for each $\boldsymbol{a} \in \mathbb{Z}^{n}$, either there is $\boldsymbol{c} \in \Sigma$ with $\langle\boldsymbol{c}, \boldsymbol{a}\rangle=0$, or $F\left(t^{\boldsymbol{a}}, z\right)$ is irreducible.

Previously, Schinzel [Sch90] proved a similar result in the same direction, for Laurent polynomials over $\mathbb{Q}$ satisfying the strong additional assumption that $F$ is not self-inversive. More recently, Fuchs, Mantova and Zannier [FMZ18, Addendum to Theorem 1.5] showed that the set $\Sigma$ can be chosen independently of the coefficients of $F$.

In the present paper we are interested in the factorization of $F_{\boldsymbol{a}}$. Our motivation is an old conjecture of Schinzel [Sch65] on the factorisation of sparse polynomial with rational coefficients (Conjecture 2.1).

This conjecture implies the statement below. A Laurent polynomial in $\mathbb{Q}\left[\boldsymbol{x}^{ \pm 1}\right]$ is cyclotomic if it can be written as a unit times the composition of a univariate cyclotomic polynomial with a monomial.

Conjecture 1.2. Let $F \in \mathbb{Q}\left[\boldsymbol{x}^{ \pm 1}\right]$. There is a finite set of matrices $\Omega \subset \mathbb{Z}^{n \times n}$ satisfying the following property. For each $\boldsymbol{a} \in \mathbb{Z}^{n}$, there are $M \in \Omega$ and $\boldsymbol{b} \in \mathbb{Z}^{n}$ with $\boldsymbol{a}=M \boldsymbol{b}$ such that if $P$ is an irreducible factor of $F\left(\boldsymbol{x}^{M}\right)$, then $P\left(t^{\boldsymbol{b}}\right)$ is either a product of cyclotomic Laurent polynomials, or an irreducible factor of $F\left(t^{a}\right)$.

Our main result in this text is the following function field analogue.

Theorem 1.3. Let $F \in \mathbb{C}\left[\boldsymbol{x}^{ \pm 1}, z^{ \pm 1}\right]$. There is a finite set of matrices $\Omega \subset \mathbb{Z}^{n \times n}$ satisfying the following property. For each $\boldsymbol{a} \in \mathbb{Z}^{n}$, there are $M \in \Omega$ and $\boldsymbol{b} \in \mathbb{Z}^{n}$ with $\boldsymbol{a}=M \boldsymbol{b}$ such that if $P$ is an irreducible factor of $F\left(\boldsymbol{x}^{M}, z\right)$, then $P\left(t^{\boldsymbol{b}}, z\right)$ is, as an element of $\mathbb{C}(t)\left[z^{ \pm 1}\right]$, either a unit or an irreducible factor of $F\left(t^{a}, z\right)$.

Moreover, we also obtain in Theorem 5.1 the function field analogue of Conjecture 2.1 .

Theorem 1.3 shows that for each $\boldsymbol{a} \in \mathbb{Z}^{n}$, there is a matrix $M$ within the finite set $\Omega \subset \mathbb{Z}^{n \times n}$ and a vector $\boldsymbol{b} \in \mathbb{Z}^{n}$ with $\boldsymbol{a}=M \boldsymbol{b}$ such that, unless $F\left(\boldsymbol{x}^{M}, z\right)=0$, the irreducible factorization

$$
F\left(\boldsymbol{x}^{M}, z\right)=\prod_{P} P(\boldsymbol{x}, z)^{e_{P}}
$$

yields the irreducible factorization in the ring $\mathbb{C}(t)\left[z^{ \pm 1}\right]$

$$
F_{\boldsymbol{a}}=\gamma \prod_{P}^{\prime} P\left(t^{\boldsymbol{b}}, z\right)^{e_{P}}
$$

for $F_{\boldsymbol{a}}$ as in 1.1 , the product being over the irreducible factors $P$ in 1.2 such that $P\left(t^{b}, z\right)$ is not a unit, and with $\gamma \in \mathbb{C}(t)\left[z^{ \pm 1}\right]^{\times}$.

Hence, the irreducible factorizations in $\mathbb{C}(t)\left[z^{ \pm 1}\right]$ of the $F_{\boldsymbol{a}}$ 's can be obtained by specializing the irreducible factorizations of the Laurent polynomials $F\left(\boldsymbol{x}^{M}, z\right)$ for a finite number of matrices $M$. These irreducible factors of the $F_{\boldsymbol{a}}$ 's are sparse, in the sense that they are all represented as the pullback of a finite number of regular functions on the $(n+1)$-dimensional torus $\mathbb{G}_{\mathrm{m}}^{n+1}$ by 2-parameter monomial maps. In particular, both the number of these irreducible factors and of their coefficients are bounded above independently of $\boldsymbol{a}$.

The proof of Theorem 1.3 relies on a variant of the aforementioned result of Zannier. To state it, we first introduce some further notation. Let $\boldsymbol{t}=\left(t_{1}, \ldots, t_{k}\right)$ be a set of 
$k$ variables. A matrix $A=\left(a_{i, j}\right)_{i, j} \in \mathbb{Z}^{n \times k}$ defines the family of $n$ monomials in the variables $\boldsymbol{t}$ given by

$$
\boldsymbol{t}^{A}=\left(\prod_{j=1}^{k} t_{j}^{a_{1, j}}, \ldots, \prod_{j=1}^{k} t_{j}^{a_{n, j}}\right) .
$$

Given $\boldsymbol{a}=\left(a_{1}, \ldots, a_{n}\right) \in \mathbb{Z}^{n}$, we can consider it as a row vector or as a column vector. Thus

$$
\boldsymbol{x}^{\boldsymbol{a}}=\prod_{j=1}^{n} x_{j}^{a_{j}} \quad \text { and } \quad t^{\boldsymbol{a}}=\left(t^{a_{1}}, \ldots, t^{a_{n}}\right) .
$$

Theorem 1.4. Let $F \in \mathbb{C}\left[\boldsymbol{x}^{ \pm 1}, z^{ \pm 1}\right] \backslash \mathbb{C}\left[\boldsymbol{x}^{ \pm 1}\right]$ be an irreducible Laurent polynomial, and $G \in \mathbb{C}\left[\boldsymbol{x}^{ \pm 1}\right]$ the coefficient of the term of highest degree in the variable $z$. There are finite subsets $\Phi \subset \mathbb{Z}^{n \times n}$ of nonsingular matrices and $\Sigma \subset \mathbb{Z}^{n}$ of nonzero vectors such that, for $\boldsymbol{a} \in \mathbb{Z}^{n}$, one of the next alternatives holds:

(1) there is $\boldsymbol{c} \in \Sigma$ such that $\langle\boldsymbol{c}, \boldsymbol{a}\rangle=0$;

(2) there is $M \in \Phi$ such that $\boldsymbol{a} \in \operatorname{im}(M)$ and $F\left(\boldsymbol{x}^{M}, z\right)$ is reducible;

(3) the Laurent polynomial $F\left(t^{a}, z\right) \in \mathbb{C}\left[t^{ \pm 1}, z^{ \pm 1}\right]$ is irreducible in $\mathbb{C}\left[t^{ \pm 1}, z^{ \pm 1}\right]_{G\left(t^{a}\right)}$.

Back to the factorization problem for sparse polynomials, it is natural to consider the more general setting of pullbacks of regular functions on $\mathbb{G}_{\mathrm{m}}^{n}$ by arbitrary monomial maps, instead of only those appearing in (1.1). Let $\boldsymbol{y}=\left(y_{1}, \ldots, y_{n}\right)$ and $\boldsymbol{t}=\left(t_{1}, \ldots, t_{k}\right)$ be groups of $n$ and $k$ variables, respectively. For a Laurent polynomial $H \in \mathbb{C}\left[\boldsymbol{y}^{ \pm 1}\right]$, consider the family of $k$-variate Laurent polynomials given by the pullback of $H$ by the monomial map $\mathbb{G}_{\mathrm{m}}^{k} \rightarrow \mathbb{G}_{\mathrm{m}}^{n}$ defined by $\boldsymbol{t} \mapsto \boldsymbol{t}^{A}$ for a matrix $A \in \mathbb{Z}^{n \times k}$, that is

$$
H_{A}=H\left(\boldsymbol{t}^{A}\right) \in \mathbb{C}\left[\boldsymbol{t}^{ \pm 1}\right] \text {. }
$$

Denote by $S$ the multiplicative subset of $\mathbb{C}\left[\boldsymbol{t}^{ \pm 1}\right]$ generated by the Laurent polynomials of the form $f\left(\boldsymbol{t}^{\boldsymbol{d}}\right)$ for $f \in \mathbb{C}\left[z^{ \pm 1}\right]$ and $\boldsymbol{d} \in \mathbb{Z}^{k}$.

We propose the following conjecture which, as explained in Remark 5.2, partially generalizes Theorem 1.3 .

Conjecture 1.5. Let $H \in \mathbb{C}\left[\boldsymbol{y}^{ \pm 1}\right]$ and $k \geq 2$. There is a finite set of matrices $\Omega \subset \mathbb{Z}^{n \times n}$ satisfying the following property. For each $A \in \mathbb{Z}^{n \times k}$, there are $N \in \Omega$ and $B \in \mathbb{Z}^{n \times k}$ with $A=N B$ such that if $P$ is an irreducible factor of $H\left(\boldsymbol{y}^{N}\right)$, then $P\left(\boldsymbol{t}^{B}\right)$ is, as an element of $\mathbb{C}\left[\boldsymbol{t}^{ \pm 1}\right]_{S}$, either a unit or an irreducible factor of $H\left(\boldsymbol{t}^{A}\right)$.

The validity of this conjecture would imply that the irreducible factors of the $H_{A}$ 's that truly depend on more than one variable, are also the pullback of a finite number of regular functions on $\mathbb{G}_{\mathrm{m}}^{n}$ by $k$-parameter monomial maps. The possible univariate irreducible factors of the $H_{A}$ 's split completely, and so they cannot be accounted from a finite number of such regular functions.

This conjecture might follow from a suitable toric analogue of the classical Bertini's theorem that we propose in Conjecture 3.13 .

Plan of the paper. In Section 2 we state Schinzel's conjecture and our function field analogue (Theorem 2.4. In Section 3 we recall some facts on fiber products and prove a variant of the Fuchs-Mantova-Zannier theorem concerning the irreducibility of pullbacks of cosets by a dominant maps $W \rightarrow \mathbb{G}_{\mathrm{m}}^{n}$ (Theorem 3.4). In Section 4 we prove Theorem 1.4, wereas in Section 5 we apply this result to prove Theorem 2.4 and then Theorem 1.3 .

Acknowledgments. We thank Pietro Corvaja, Qing Liu, Vincenzo Mantova, Juan Carlos Naranjo and Umberto Zannier for useful conversations. We also thank the 
anonymous referee for his/her useful comments. Part of this work was done while the authors met the Universitat de Barcelona and the Université de Caen. We thank these institutions for their hospitality.

\section{A conjecture of Schinzel and its function field analogue}

In [Sch65], Schinzel proposed the conjecture below on the factorization of univariate polynomials over $\mathbb{Q}$.

Conjecture 2.1. Let $F \in \mathbb{Q}\left[\boldsymbol{x}^{ \pm 1}\right]$ be a non-cyclotomic irreducible Laurent polynomial. There are finite sets $\Omega^{0} \subset \mathbb{Z}^{n \times n}$ of nonsingular matrices and $\Gamma \subset \mathbb{Z}^{n}$ of nonzero vectors satisfying the following property. Let $\boldsymbol{a} \in \mathbb{Z}^{n}$; then one of the next conditions holds:

(1) there is $\boldsymbol{c} \in \Gamma$ verifying $\langle\boldsymbol{c}, \boldsymbol{a}\rangle=0$;

(2) there are $M \in \Omega^{0}$ and $\boldsymbol{b} \in \mathbb{Z}^{n}$ with $\boldsymbol{a}=M \boldsymbol{b}$ such that if

$$
F\left(\boldsymbol{x}^{M}\right)=\prod_{P} P^{e_{P}}
$$

is the irreducible factorization of $F\left(\boldsymbol{x}^{M}\right)$, then

$$
\frac{F\left(t^{\boldsymbol{a}}\right)}{\operatorname{cyc}\left(F\left(t^{\boldsymbol{a}}\right)\right.}=\prod_{P}\left(\frac{P\left(t^{\boldsymbol{b}}\right)}{\operatorname{cyc}\left(P\left(t^{\boldsymbol{b}}\right)\right)}\right)^{e_{P}}
$$

is the irreducible factorization of $F\left(t^{\boldsymbol{a}}\right) / \operatorname{cyc}\left(F\left(t^{\boldsymbol{a}}\right)\right)$.

For the validity of this statement, in its condition (2) it is necessary to take out the

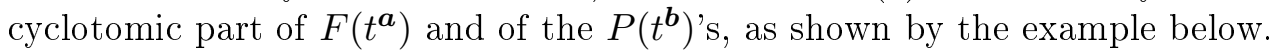

Example 2.2. Set $F=x_{1}+x_{2}-2 \in \mathbb{Q}\left[x_{1}^{ \pm 1}, x_{2}^{ \pm 1}\right]$. Let $\boldsymbol{a} \in \mathbb{Z}^{2}$ and choose a nonsingular matrix $M \in \mathbb{Z}^{2 \times 2}$ and a vector $\boldsymbol{b} \in \mathbb{Z}^{2}$ with $\boldsymbol{a}=M \boldsymbol{b}$. We have that

$$
F\left(\boldsymbol{x}^{M}\right)=x_{1}^{m_{1,1}} x_{2}^{m_{1,2}}+x_{1}^{m_{2,1}} x_{2}^{m_{2,2}}-2
$$

is irreducible, and so $P:=F\left(\boldsymbol{x}^{M}\right)$ is the only irreducible factor of this Laurent polynomial. However, $t-1$ divides $F\left(t^{\boldsymbol{a}}\right)=P\left(t^{\boldsymbol{b}}\right)$, and so these univariate Laurent polynomials are not irreducible, unless we divide them by this cyclotomic factor.

Schinzel proved this conjecture when $n=1$ in loc. cit. and, under the restrictive hypothesis that $F$ is not self-inversive, when $n \geq 2$ [Sch70], see also [Sch00, $\S 6.2]$. The general case when $n \geq 2$ remains open.

In Section 5, we prove the function field analogue for Laurent polynomials over the field $\mathbb{C}(z)$ in Theorem 2.4 below. An element of $\mathbb{C}(z)\left[\boldsymbol{x}^{ \pm 1}\right]$ is constant if it lies in $\mathbb{C}\left[\boldsymbol{x}^{ \pm 1}\right]$, up to a scalar factor in $\mathbb{C}(z)^{\times}$. The constant part of a Laurent polynomial $F \in \mathbb{C}(z)\left[\boldsymbol{x}^{ \pm 1}\right] \backslash\{0\}$, denoted by $\operatorname{ct}(F)$, is defined as its maximal constant factor. This constant part is well-defined up to a unit of $\mathbb{C}(z)\left[\boldsymbol{x}^{ \pm 1}\right]$.

Remark 2.3. The analogy between cyclotomic Laurent polynomials over $\mathbb{Q}$ and irreducible constant Laurent polynomials over $\mathbb{C}(z)$ stems from height theory. Let $\mathbb{K}$ denote either $\mathbb{Q}$ or $\mathbb{C}(z)$, and $h$ the canonical height function on subvarieties of the torus $\mathbb{G}_{\mathrm{m}, \mathbb{K}}^{n}$, induced by the standard inclusion $\mathbb{G}_{\mathrm{m}, \mathbb{K}}^{n} \hookrightarrow \mathbb{P}_{\mathbb{K}}^{n}$.

Let $F \in \mathbb{K}\left[\boldsymbol{x}^{ \pm 1}\right]$ be an irreducible Laurent polynomial defining a hypersurface $V(F)$ of $\mathbb{G}_{\mathrm{m}}^{n}$. Then the condition that $\mathrm{h}(V(F))=0$ is equivalent to the fact that $F$ is cyclotomic when $\mathbb{K}=\mathbb{Q}$, and to the fact that $F$ is constant when $\mathbb{K}=\mathbb{C}(z)$.

Theorem 2.4. Let $F \in \mathbb{C}(z)\left[\boldsymbol{x}^{ \pm 1}\right]$ be a non-constant irreducible Laurent polynomial. There are finite sets $\Omega^{0} \subset \mathbb{Z}^{n \times n}$ of nonsingular matrices and $\Gamma \subset \mathbb{Z}^{n}$ of nonzero vectors satisfying the following property. Let $\boldsymbol{a} \in \mathbb{Z}^{n}$; then one of the next conditions holds: 
(1) there is $\boldsymbol{c} \in \Gamma$ verifying $\langle\boldsymbol{c}, \boldsymbol{a}\rangle=0$;

(2) there are $M \in \Omega^{0}$ and $\boldsymbol{b} \in \mathbb{Z}^{n}$ with $\boldsymbol{a}=M \boldsymbol{b}$ such that if

$$
F\left(\boldsymbol{x}^{M}\right)=\prod_{P} P^{e_{P}}
$$

is the irreducible factorization of $F\left(\boldsymbol{x}^{M}\right)$, then

$$
\frac{F\left(t^{\boldsymbol{a}}\right)}{\operatorname{ct}\left(F\left(t^{\boldsymbol{a}}\right)\right)}=\prod_{P}\left(\frac{P\left(t^{\boldsymbol{b}}\right)}{\operatorname{ct}\left(P\left(t^{\boldsymbol{b}}\right)\right)}\right)^{e_{P}}
$$

is the irreducible factorization of $F\left(t^{\boldsymbol{a}}\right) / \operatorname{ct}\left(F\left(t^{\boldsymbol{a}}\right)\right)$.

Similarly as for Conjecture 2.1, for the validity this statement it is is necessary to take out in its condition (2) the constant part of $F\left(t^{\boldsymbol{a}}\right)$ and of the $P\left(t^{\boldsymbol{b}}\right)$ 's.

Example 2.5. Set $F=x_{1}+z x_{2}-z-1 \in \mathbb{C}(z)\left[x_{1}^{ \pm 1}, x_{2}^{ \pm 1}\right]$. Let $\boldsymbol{a} \in \mathbb{Z}^{2}$ and choose $M \in \mathbb{Z}^{2 \times 2}$ nonsingular and $\boldsymbol{b} \in \mathbb{Z}^{2}$ with $\boldsymbol{a}=M \boldsymbol{b}$. We have that

$$
F\left(\boldsymbol{x}^{M}\right)=x_{1}^{m_{1,1}} x_{2}^{m_{1,2}}+z x_{1}^{m_{2,1}} x_{2}^{m_{2,2}}-z-1
$$

is irreducible, and so $P:=F\left(\boldsymbol{x}^{M}\right)$ is its only irreducible factor. Again, $t-1$ divides $F\left(t^{\boldsymbol{a}}\right)=P\left(t^{\boldsymbol{b}}\right)$, and so these univariate Laurent polynomials are not irreducible, unless we divide them by a suitable constant factor.

Remark 2.6. The validity of Schinzel's conjecture 2.1 would imply that of Conjecture 1.5, in the same way that Theorem 2.4 implies Theorem 1.3, as explained in Section 5.

\section{A VARIant of Zannier's toric Bertini's theorem}

Zannier proved in [Zan10] an analogue of Bertini's theorem for covers, where the subtori of $\mathbb{G}_{\mathrm{m}}^{n}$ replaced the linear subspaces in the classical version of this theorem. This result was precised and generalized (with a completely different proof) by Fuchs, Mantova and Zannier to include fibers of arbitrary cosets of subtori [FMZ18, Theorem 1.5] and to obtain a more uniform result.

As before, let $\boldsymbol{x}=\left(x_{1}, \ldots, x_{n}\right)$ be a set of $n$ variables and denote by $\mathbb{G}_{\mathrm{m}}^{n}=$ $\operatorname{Spec}\left(\mathbb{C}\left[\boldsymbol{x}^{ \pm 1}\right]\right)$ the $n$-dimensional torus over $\mathbb{C}$.

Let $W$ be a variety, that is, a reduced separated scheme of finite type over $\mathbb{C}$. We assume that $W$ is irreducible and quasiprojective of dimension $n \geq 0$, and equipped with a dominant (regular) map

$$
\pi: W \longrightarrow \mathbb{G}_{\mathrm{m}}^{n}
$$

of degree $e \geq 1$ that is finite onto its image. Given an isogeny $\lambda$ of $\mathbb{G}_{\mathrm{m}}^{n}$, that is, an endomorphism of $\mathbb{G}_{\mathrm{m}}^{n}$ with finite kernel, we denote by $\lambda^{*} W$ the fibered product $\mathbb{G}_{\mathrm{m}}^{n} \times_{\lambda, \pi} W$, and by

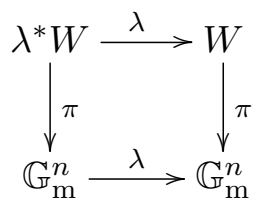

the corresponding fibered product square.

Definition 3.1. The map $\pi$ satisfies the property $P B$ (pullback) if, for every isogeny $\lambda$ of $\mathbb{G}_{\mathrm{m}}^{n}$, we have that $\lambda^{*} W$ is an irreducible variety. 
By [Zan10, Proposition 2.1], it is enough to test this condition for $\lambda=[e]$, the multiplication map of $\mathbb{G}_{\mathrm{m}}^{n}$ by the integer $e=\operatorname{deg}(\pi)$.

The aforementioned result by Fuchs, Mantova and Zannier can be stated as follows.

Theorem 3.2. Let $W$ be an irreducible quasiprojective variety of dimension $n$ and $\pi: W \rightarrow \mathbb{G}_{\mathrm{m}}^{n}$ a dominant map that is finite onto its image and that satisfies the property $P B$. There is a finite union $\mathcal{E}$ of proper subtori of $\mathbb{G}_{\mathrm{m}}^{n}$ such that, for every subtorus $T \subset \mathbb{G}_{\mathrm{m}}^{n}$ not contained in $\mathcal{E}$ and every point $p \in \mathbb{G}_{\mathrm{m}}^{n}(\mathbb{C})=\left(\mathbb{C}^{\times}\right)^{n}$, we have that $\pi^{-1}(p \cdot T)$ is an irreducible subvariety of $W$.

When the property PB is not verified, the conclusion of this theorem does not necessarily hold because the map $\pi$ factors through a nontrivial isogeny, as it was already pointed out in [Zan10].

Example 3.3. Let $F=z^{2}-x_{1} x_{2}^{2} \in \mathbb{C}\left[x_{1}^{ \pm 1}, x_{2}^{ \pm 1}, z^{ \pm 1}\right]$, set $W$ be the torus $V(F) \subset \mathbb{G}_{\mathrm{m}}^{3}$ and consider the isogeny

$$
\pi: W \longrightarrow \mathbb{G}_{\mathrm{m}}^{2}
$$

defined by $\pi\left(x_{1}, x_{2}, z\right)=\left(x_{1}, x_{2}\right)$ for $\left(x_{1}, x_{2}, z\right) \in W$.

The variety $W$ is irreducible and, since $F$ is monic in $z$, the map $\pi$ is finite. However, it does not satisfy the property $\mathrm{PB}$, since for the isogeny $\lambda$ of $\mathbb{G}_{\mathrm{m}}^{2}$ defined by $\lambda\left(x_{1}, x_{2}\right)=$ $\left(x_{1}^{2}, x_{2}\right)$,

$$
\lambda^{*} W \simeq V\left(z-x_{1} x_{2}\right) \cup V\left(z+x_{1} x_{2}\right)
$$

and so this pullback is reducible.

Indeed, this map does neither satisfy the conclusion of Theorem 3.2 given $\left(a_{1}, a_{2}\right) \in$ $\mathbb{Z}^{2} \backslash\{(0,0)\}$ with $a_{1}$ even, let $T \subset \mathbb{G}_{\mathrm{m}}^{2}$ be the 1 -dimensional subtorus given as the image of the map $t \mapsto\left(t^{a_{1}}, t^{a_{2}}\right)$. Then

$$
\pi^{-1}(T)=V\left(z-t^{a_{1} / 2} t^{a_{2}}\right) \cup V\left(z+t^{a_{1} / 2} t^{a_{2}}\right),
$$

and so this fiber is not irreducible.

Here we need a variant of Theorem 3.2 that can be also applied in the situation when the map $\pi$ does not verify the property PB. In this more general situation, the conclusion of that theorem does not necessarily hold. Instead, this conclusion is replaced by an alternative that "explains" the possibility that a fiber is reducible by its factorization through a reducible pullback of the variety $W$ by an isogeny of $\mathbb{G}_{\mathrm{m}}^{n}$ within a finite set.

Theorem 3.4. Let $W$ be an irreducible quasiprojective variety of dimension $n$ and $\pi: W \rightarrow \mathbb{G}_{\mathrm{m}}^{n}$ a dominant map that is finite onto its image. There is a finite union $\mathcal{E}$ of proper subtori of $\mathbb{G}_{\mathrm{m}}^{n}$ and a finite set $\Lambda$ of isogenies of $\mathbb{G}_{\mathrm{m}}^{n}$ such that, for each subtorus $T \subset \mathbb{G}_{\mathrm{m}}^{n}$ and each point $p \in \mathbb{G}_{\mathrm{m}}^{n}(\mathbb{C})=\left(\mathbb{C}^{\times}\right)^{n}$, one of the next conditions holds:

(1) $T \subseteq \mathcal{E}$

(2) there is $\lambda \in \Lambda$ with $\lambda^{*} W$ reducible and a subtorus $T^{\prime} \subset \mathbb{G}_{\mathrm{m}}^{n}$ with $\lambda$ inducing an isomorphism $T^{\prime} \rightarrow T$;

(3) $\pi^{-1}(p \cdot T)$ is irreducible.

Remark 3.5. When the condition (2) above is satisfied, there is a diagram

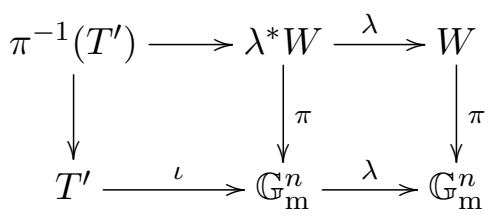


with $\lambda^{*} W$ reducible and $\lambda: T^{\prime} \rightarrow T$ an isomorphism, and where $\iota$ denotes the inclusion of the subtorus $T^{\prime}$ into $\mathbb{G}_{\mathrm{m}}^{n}$.

Both inner squares in this diagram are fibered products, and so is the outer square. This implies that the fibers $\pi^{-1}(T)$ and $\pi^{-1}\left(T^{\prime}\right)$ are isomorphic. Thus $\pi^{-1}(T)$ can be identified with the fiber of a subtorus for the reducible cover $\pi: \lambda^{*} W \rightarrow \mathbb{G}_{\mathrm{m}}^{n}$, and so this fiber is expected to be reducible as well.

Example 3.6. We keep the notation from Example 3.3. In particular, $F=z^{2}-x_{1} x_{2}^{2} \in$ $\mathbb{C}\left[x_{1}^{ \pm 1}, x_{2}^{ \pm 1}, z^{ \pm 1}\right], W$ the torus $V(F) \subset \mathbb{G}_{\mathrm{m}}^{3}$, and $\pi: W \rightarrow \mathbb{G}_{\mathrm{m}}^{2}$ the isogeny defined by $\pi\left(x_{1}, x_{2}, z\right)=\left(x_{1}, x_{2}\right)$.

Let $\left(a_{1}, a_{2}\right) \in \mathbb{Z}^{2} \backslash\{(0,0)\}$ with $a_{1}$ even, and set $T \subset \mathbb{G}_{\mathrm{m}}^{2}$ for the 1-dimensional subtorus given as the image of the map $t \mapsto\left(t^{a_{1}}, t^{a_{2}}\right)$. These vectors satisfy the condition (2) in Theorem 3.4 for the isogeny $\lambda: \mathbb{G}_{\mathrm{m}}^{2} \rightarrow \mathbb{G}_{\mathrm{m}}^{2}$ defined by

$$
\lambda\left(x_{1}, x_{2}\right)=\left(x_{1}^{2}, x_{2}\right) .
$$

Indeed, $\lambda^{*} W$ is reducible, and this isogeny induces an isomorphism $T^{\prime} \rightarrow T$ with the subtorus $T^{\prime} \subset \mathbb{G}_{\mathrm{m}}^{2}$ given as the image of the map $t \mapsto\left(t^{a_{1} / 2}, t^{a_{2}}\right)$.

We prove this theorem by reducing it to the previous toric Bertini's theorem, through a variation (Proposition 3.8) of a factorization result for rational maps from [Zan10]. We give the proof after some auxiliary results. We first study the reducibility of pullbacks of varieties with respect to isogenies of tori.

Lemma 3.7. Let $\pi: W \rightarrow X$ be a map of varieties and $\lambda: X \rightarrow X$ an étale map. Then $X \times_{\lambda, \pi} W$ is a variety.

In particular, for a map $\pi: W \rightarrow \mathbb{G}_{\mathrm{m}}^{n}$ and an isogeny $\lambda$ of $\mathbb{G}_{\mathrm{m}}^{n}$, we have that $\lambda^{*} W$ is a variety.

Proof. Since $\lambda: X \rightarrow X$ is étale, the map

$$
\lambda: X \times_{\lambda, \pi} W \longrightarrow W
$$

is also étale, because of the invariance of this property under base change [Har77, Chapter IV, Proposition 10.1(b)]. By [Har77, Chapter IV, Exercise 10.4], this implies that, for every closed point $q \in X \times_{\lambda, \pi} W$ and $p:=\lambda(q) \in W$, the induced map of completed local rings

$$
\widehat{\mathcal{O}}_{p} \longrightarrow \widehat{\mathcal{O}}_{q}
$$

is an isomorphism. Since $W$ is a variety, the local ring $\mathcal{O}_{p}$ is reduced and, by a theorem of Chevalley [ZS75, §8.13], the completion $\widehat{\mathcal{O}}_{p}$ is reduced too.

By the isomorphism in (3.3), the completed ring $\widehat{\mathcal{O}}_{q}$ is reduced. Since this is the completion of a ring with respect to a maximal ideal, the map $\mathcal{O}_{q} \rightarrow \widehat{\mathcal{O}}_{q}$ is injective, and so the local ring $\mathcal{O}_{q}$ is also reduced. Since the condition of being reduced is local, this implies that $X \times_{\lambda, \pi} W$ is a variety.

The last statement comes from the fact that the isogenies of algebraic groups over $\mathbb{C}$ are étale maps.

Thanks to this result, $\lambda^{*} W$ can be identified with its underlying algebraic subset in the Cartesian product $\mathbb{G}_{\mathrm{m}}^{n}(\mathbb{C}) \times W(\mathbb{C})$, namely

$$
\lambda^{*} W=\left\{(p, w) \in \mathbb{G}_{\mathrm{m}}^{n}(\mathbb{C}) \times W(\mathbb{C}) \mid \lambda(p)=\pi(w)\right\} .
$$

Hence, $\lambda^{*} W$ is irreducible if and only if this algebraic subset is irreducible. In particular, the map $\pi$ satisfies the property PB if and only if for every isogeny $\lambda$ of $\mathbb{G}_{\mathrm{m}}^{n}$, the pullback $\lambda^{*} W$ has a single irreducible component. 
The following proposition is implicit in the proof of [Zan10, Proposition 2.1].

Proposition 3.8. Let $\pi: W \rightarrow \mathbb{G}_{\mathrm{m}}^{n}$ be a map from an irreducible variety $W$, and $\lambda$ an isogeny of $\mathbb{G}_{\mathrm{m}}^{n}$. The following conditions are equivalent:

(1) the pullback $\lambda^{*} W$ is reducible;

(2) there is a factorization $\lambda=\mu \circ \tau$ with $\mu, \tau$ isogenies of $\mathbb{G}_{\mathrm{m}}^{n}$ such that $\mu$ is not an isomorphism, and a map $\rho: W \rightarrow \mathbb{G}_{\mathrm{m}}^{n}$ such that $\pi=\mu \circ \rho$.

In other terms, the condition (2) in the proposition above amounts to the existence of the commutative diagram extending 3.1 of the form

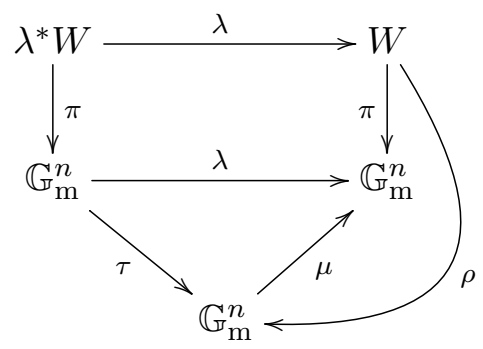

Proof. Suppose that the condition (2) holds. In this case, for $p \in \mathbb{G}_{\mathrm{m}}^{n}(\mathbb{C})$ and $w \in$ $W(\mathbb{C})$, the fact that $\lambda(p)=\pi(w)$ is equivalent to $\mu(\tau(p))=\mu(\rho(w))$, and so this holds if and only if there is $\zeta \in \operatorname{ker}(\mu)$ with $\tau(p)=\zeta \cdot \rho(w)$. From (3.4), the pullback decomposes into disjoints subvarieties as

$$
\lambda^{*} W=\bigcup_{\zeta \in \operatorname{ker}(\mu)} \mathbb{G}_{\mathrm{m}}^{n} \times_{\tau, \zeta \cdot \rho} W .
$$

Since $\mu$ is not an isomorphism, this pullback is reducible, giving the condition (1).

Conversely, suppose that the condition (1) holds. Then $\lambda^{*} W$ has a decomposition into irreducible components

$$
\lambda^{*} W=\bigcup_{i=1}^{k} U_{i}
$$

with $k \geq 2$. Similarly as in $(3.2)$, the map $\lambda^{*} W \rightarrow W$ is étale, and so the $U_{i}$ 's are disjoint. Since $\lambda$ is an isogeny, the map $\lambda^{*} W \rightarrow W$ is also finite.

The finite subgroup $\operatorname{ker}(\lambda)$ of $\mathbb{G}_{\mathrm{m}}^{n}(\mathbb{C})$ acts on $\lambda^{*} W$ via the maps $(p, w) \mapsto(\zeta \cdot p, w)$ for $\zeta \in \operatorname{ker}(\lambda)$, and this action respects the fibers of $\lambda$. The action is transitive on the fibers, and so it is also transitive on the $U_{i}$ 's.

Let $H \subset \operatorname{ker}(\lambda)$ be the stabilizer of the irreducible component $U_{1}$, and $U_{1} / H$ the quotient variety. We have that $H$ acts on $U_{1}$ transitively on the fibers and without fixed points. The induced map

$$
U_{1} / H \longrightarrow W
$$

is a finite étale map of degree 1, and so it is an isomorphism [Mum88, §III.10, Proposition 2].

Then we define the map $\rho: W \rightarrow \mathbb{G}_{\mathrm{m}}^{n}$ as the map obtained from the quotient map $U_{1} / H \rightarrow \mathbb{G}_{\mathrm{m}}^{n} / H$ and the identifications $U_{1} / H \simeq W$ and $\mathbb{G}_{\mathrm{m}}^{n} / H \simeq \mathbb{G}_{\mathrm{m}}^{n}$. In concrete terms and identifying $\mathbb{G}_{\mathrm{m}}^{n} / H \simeq \mathbb{G}_{\mathrm{m}}^{n}$, this map is defined, for $w \in W$, as $\rho(w)=\tau(p \cdot H)$ for any $p \in \mathbb{G}_{\mathrm{m}}^{n}$ such that $(p, w) \in U_{1}$.

Both $\mathbb{G}_{\mathrm{m}}^{n} / H$ and $\mathbb{G}_{\mathrm{m}}^{n} / \operatorname{ker}(\lambda)$ are isomorphic to $\mathbb{G}_{\mathrm{m}}^{n}$, and so there is a factorization

$$
\lambda=\mu \circ \tau,
$$


with $\tau$ and $\mu$ corresponding to the projections $\mathbb{G}_{\mathrm{m}}^{n} \rightarrow \mathbb{G}_{\mathrm{m}}^{n} / H$ and $\mathbb{G}_{\mathrm{m}}^{n} / H \rightarrow \mathbb{G}_{\mathrm{m}}^{n} / \operatorname{ker}(\lambda)$, respectively. For $w \in W$ and $(p, w) \in U_{1}$, we have that $\mu \circ \rho(w)=\mu \circ \tau(p)=\pi(w)$. Since the action of $\operatorname{ker}(\lambda)$ on the $U_{i}$ 's is transitive and $k \geq 2$, we have that $H \neq \operatorname{ker}(\lambda)$ and so $\mu$ is not an isomorphism, giving the condition (2).

Remark 3.9. By this proof, if $\lambda^{*} W$ is reducible, then the number of its irreducible components is equal to the maximum of the quantity $\operatorname{deg}(\mu)=[\operatorname{ker}(\lambda): H]$ over all possible maps $\rho$ as in the condition (2).

The next result allows to factorize the dominant map $\pi: W \rightarrow \mathbb{G}_{\mathrm{m}}^{n}$ as a map satisfying the property PB followed by an isogeny. It is a variant of [Zan10, Proposition 2.1], that states a similar property for dominant rational maps.

Corollary 3.10. Let $W$ be an irreducible variety of dimension $n$ and $\pi: W \rightarrow \mathbb{G}_{\mathrm{m}}^{n}$ a dominant map. There are a map $\rho: W \rightarrow \mathbb{G}_{\mathrm{m}}^{n}$ satisfying the property $P B$ and an isogeny $\lambda$ of $\mathbb{G}_{\mathrm{m}}^{n}$ with $\pi=\lambda \circ \rho$.

Proof. Choose $\rho$ as a map $W \rightarrow \mathbb{G}_{\mathrm{m}}^{n}$ of minimal degree among those that give a factorization of the form $\pi=\lambda \circ \rho$ with $\lambda$ an isogeny of $\mathbb{G}_{\mathrm{m}}^{n}$.

Suppose that there is a further isogeny $\nu$ such that $\nu^{*} W=\mathbb{G}_{\mathrm{m}}^{n} \times_{\nu, \rho} W$ is reducible. By Proposition 3.8, there would be an isogeny $\mu$ that is not an isomorphism and a $\operatorname{map} \rho^{\prime}: W \rightarrow \mathbb{G}_{\mathrm{m}}^{n}$ with $\rho=\mu \circ \rho^{\prime}$. Hence

$$
\pi=\lambda \circ \rho=(\lambda \circ \mu) \circ \rho^{\prime} \quad \text { and } \quad \operatorname{deg}(\rho)=\# \operatorname{ker}(\mu) \cdot \operatorname{deg}\left(\rho^{\prime}\right)>\operatorname{deg}\left(\rho^{\prime}\right) .
$$

By construction, this is not possible. Hence $\nu^{*} W$ is irreducible for every isogeny $\nu$ of $\mathbb{G}_{\mathrm{m}}^{n}$, and so $\rho$ satisfies the property PB.

The next result gives a criterion to detect if the inclusion of a subtorus can be factored through a given isogeny as in Proposition 3.8 2).

Lemma 3.11. Let $T \subset \mathbb{G}_{\mathrm{m}}^{n}$ be a subtorus and $\lambda$ an isogeny of $\mathbb{G}_{\mathrm{m}}^{n}$. The following conditions are equivalent:

(1) there is a subtorus $T^{\prime} \subset \mathbb{G}_{\mathrm{m}}^{n}$ such that $\lambda$ induces an isomorphism $T^{\prime} \rightarrow T$;

(2) $\lambda^{-1}(T)$ is the union of $\operatorname{deg}(\lambda)$ distinct torsion cosets.

Proof. First suppose that $\lambda^{-1}(T)$ is the union of $\operatorname{deg}(\lambda)=\# \operatorname{ker}(\lambda)$ distinct torsion cosets, and denote by $T^{\prime}$ the one that contains the neutral element. Then $T^{\prime}$ is a subtorus and $T^{\prime} \cap \operatorname{ker}(\lambda)=\{1\}$. It follows that $\left.\lambda\right|_{T^{\prime}}: T^{\prime} \rightarrow T$ is an isogeny of degree 1 and hence an isomorphism, giving the first condition.

Conversely, let $T^{\prime} \subset \mathbb{G}_{\mathrm{m}}^{n}$ be a subtorus such that $\left.\lambda\right|_{T^{\prime}}: T^{\prime} \rightarrow T$ is an isomorphism. Then

$$
\lambda^{-1}(T)=\operatorname{ker}(\lambda) \cdot T^{\prime} .
$$

Since $T^{\prime} \cap \operatorname{ker}(\lambda)=\{1\}$, this fiber is the union of $\# \operatorname{ker}(\lambda)=\operatorname{deg}(\lambda)$ distinct torsion cosets, giving the second condition.

Proof of Theorem 3.4. By Corollary 3.10, there are a map $\rho: W \rightarrow \mathbb{G}_{\mathrm{m}}^{n}$ satisfying the property $\mathrm{PB}$ and an isogeny $\lambda$ of $\mathbb{G}_{\mathrm{m}}^{n}$ with $\pi=\lambda \circ \rho$.

For each subgroup $H$ of $\operatorname{ker}(\lambda)$, both $\mathbb{G}_{\mathrm{m}}^{n} / H$ and $\mathbb{G}_{\mathrm{m}}^{n} / \operatorname{ker}(\lambda)$ are isomorphic to $\mathbb{G}_{\mathrm{m}}^{n}$, and we consider then a factorization

$$
\lambda=\mu_{H} \circ \tau_{H}
$$

with $\tau_{H}$ and $\mu_{H}$ corresponding to the projections $\mathbb{G}_{\mathrm{m}}^{n} \rightarrow \mathbb{G}_{\mathrm{m}}^{n} / H$ and $\mathbb{G}_{\mathrm{m}}^{n} / H \rightarrow$ $\mathbb{G}_{\mathrm{m}}^{n} / \operatorname{ker}(\lambda)$, respectively. We set $\Lambda$ as the finite set of isogenies of $\mathbb{G}_{\mathrm{m}}^{n}$ of the form $\mu_{H}$ as above, for a proper subgroup $H$ of $\operatorname{ker}(\lambda)$. 
Since $\rho: W \rightarrow \mathbb{G}_{\mathrm{m}}^{n}$ satisfies the property PB, by [FMZ18, Theorem 1.5] there is a finite union $\mathcal{E}^{\prime}$ of proper subtori of $\mathbb{G}_{\mathrm{m}}^{n}$ such that, for every subtorus $T$ of $\mathbb{G}_{\mathrm{m}}^{n}$ not contained in $\mathcal{E}^{\prime}$ and every point $p \in \mathbb{G}_{\mathrm{m}}^{n}(\mathbb{C})$, the fiber $\rho^{-1}(p \cdot T)$ is irreducible. Set $\mathcal{E}=\lambda\left(\mathcal{E}^{\prime}\right)$.

We next show that the pair $(\Lambda, \mathcal{E})$ satisfies the requirements of Theorem 3.4 Let $T$ be a subtorus of $\mathbb{G}_{\mathrm{m}}^{n}$ that is not contained in $\mathcal{E}$ and write $\lambda^{-1}(T)=\bigcup_{i=1}^{k} T_{i}$ as a disjoint union of torsion cosets $T_{i}$ of $\mathbb{G}_{\mathrm{m}}^{n}$.

When $k=1$, we have that $\lambda^{-1}(T)=T_{1}$ is a subtorus of $\mathbb{G}_{\mathrm{m}}^{n}$ that is not contained in $\mathcal{E}$. Hence, $\pi^{-1}(T)=\rho^{-1}\left(T_{1}\right)$ is irreducible.

Otherwise, $k \geq 2$. Let $H \subset \operatorname{ker}(\lambda)$ be the stabilizer of the (unique) subtori in this decomposition, say $T_{1}$. This is a proper subgroup, because $\operatorname{ker}(\lambda)$ acts transitively on this collection of torsion cosets and $k \geq 2$.

Consider the factorization $\lambda=\mu_{H} \circ \tau_{H}$ as in 3.5. Then $\mu_{H} \in \Lambda$ and $\mu_{H}^{-1}(T)$ splits as an union of $k=[\operatorname{ker}(\lambda): H]=\operatorname{deg}\left(\mu_{H}\right)$ distinct torsion cosets. By Lemma 3.11. $\mu_{H}$ induces an isomorphism between a subtorus $T^{\prime}$ of $\mathbb{G}_{\mathrm{m}}^{n}$ and $T$. Moreover, Proposition 3.8 2) applied to the map $\tau_{H} \circ \rho$ and the isogeny $\mu_{H}$ shows that the pullback $\mu_{H}^{*} W$ is reducible, completing the proof.

It seems interesting to extend these results to maps that are not necessarily dominant. In this direction, we propose the following conjectural extension of the FuchsMantova-Zannier theorem 3.2. It can be seen as a toric analogue of the classical Bertini's theorem as stated in [Jou83, Théorème 6.3(3)].

Conjecture 3.12. Let $W$ be an irreducible quasiprojective variety and $\varphi: W \rightarrow \mathbb{G}_{\mathrm{m}}^{n}$ a map that is finite onto its image and satisfies the property $P B$. There is a finite union $\mathcal{E}$ of proper subtori of $\mathbb{G}_{\mathrm{m}}^{n}$ such that, for every subtorus $T$ of $\mathbb{G}_{\mathrm{m}}^{n}$ with

$$
\operatorname{dim}(T) \geq \operatorname{codim}(\overline{\varphi(W)})+1
$$

that is not contained in $\mathcal{E}$ and every point $p \in \mathbb{G}_{\mathrm{m}}^{n}(\mathbb{C})$, we have that $\varphi^{-1}(p \cdot T)$ is an irreducible subvariety of $W$.

Similarly, we propose the following conjectural extension of Theorem 3.4

Conjecture 3.13. Let $W$ be an irreducible quasiprojective variety and $\varphi: W \rightarrow \mathbb{G}_{\mathrm{m}}^{n}$ a map that is finite onto its image. There is a finite union $\mathcal{E}$ of proper subtori of $\mathbb{G}_{\mathrm{m}}^{n}$ and a finite set $\Lambda$ of isogenies of $\mathbb{G}_{\mathrm{m}}^{n}$ such that, for each subtorus $T \subset \mathbb{G}_{\mathrm{m}}^{n}$ with

$$
\operatorname{dim}(T) \geq \operatorname{codim}(\overline{\varphi(W)})+1
$$

and each point $p \in \mathbb{G}_{\mathrm{m}}^{n}(\mathbb{C})$, one of the next conditions holds:

(1) $T \subseteq \mathcal{E}$;

(2) there is $\lambda \in \Lambda$ with $\lambda^{*} W$ reducible and a subtorus $T^{\prime} \subset \mathbb{G}_{\mathrm{m}}^{n}$ with $\lambda$ inducing an isomorphism $T^{\prime} \rightarrow T$

(3) $\varphi^{-1}(p \cdot T)$ is irreducible.

\section{Pullbacks of Laurent polynomials by monomial maps}

We next prove Theorem 1.4 stated in the introduction. To this end, we first recall some notation and introduce some auxiliary results.

Let $\boldsymbol{t}=\left(t_{1}, \ldots, t_{k}\right)$ be a set of $k$ variables. A matrix $A=\left(a_{i, j}\right)_{i, j} \in \mathbb{Z}^{n \times k}$ defines the family of $n$ monomials in the variables $\boldsymbol{t}$ given by

$$
\boldsymbol{t}^{A}=\left(\prod_{j=1}^{k} t_{j}^{a_{1, j}}, \ldots, \prod_{j=1}^{k} t_{j}^{a_{n, j}}\right) .
$$


The rule $\boldsymbol{t} \mapsto \boldsymbol{t}^{A}$ defines a $k$-parameter monomial map $\mathbb{G}_{\mathrm{m}}^{k} \rightarrow \mathbb{G}_{\mathrm{m}}^{n}$. This is a group morphism and indeed, every group morphism from $\mathbb{G}_{\mathrm{m}}^{k}$ to $\mathbb{G}_{\mathrm{m}}^{n}$ is of this form. The isogenies of $\mathbb{G}_{\mathrm{m}}^{n}$ correspond to the nonsingular matrices of $\mathbb{Z}^{n \times n}$.

Given $\boldsymbol{a}=\left(a_{1}, \ldots, a_{n}\right) \in \mathbb{Z}^{n}$, we can consider it as a row vector, that is, as a matrix in $\mathbb{Z}^{1 \times n}$. In this case,

$$
\boldsymbol{x}^{\boldsymbol{a}}=\prod_{j=1}^{n} x_{j}^{a_{j}}
$$

is an $n$-variate monomial. Row vectors give characters of $\mathbb{G}_{\mathrm{m}}^{n}$, that is, group morphisms $\mathbb{G}_{\mathrm{m}}^{n} \rightarrow \mathbb{G}_{\mathrm{m}}$. When $\boldsymbol{a}$ is primitive, the kernel of its associated character is a subtorus of $\mathbb{G}_{\mathrm{m}}^{n}$ of codimension 1 , and every such subtorus arises in this way.

Else, we can consider $\boldsymbol{a}$ as a column vector, that is, as a matrix in $\mathbb{Z}^{n \times 1}$. Then

$$
t^{\boldsymbol{a}}=\left(t^{a_{1}}, \ldots, t^{a_{n}}\right)
$$

is a collection of $n$ univariate monomials in a variable $t$. Column vectors give group morphisms $\mathbb{G}_{\mathrm{m}} \rightarrow \mathbb{G}_{\mathrm{m}}^{n}$. When $\boldsymbol{a} \neq 0$, the image of such a morphims is a subtorus of $\mathbb{G}_{\mathrm{m}}^{n}$ of dimension 1 , that we denote by $T_{\boldsymbol{a}}$. When $\boldsymbol{a}$ is primitive, the associated group morphism $\mathbb{G}_{\mathrm{m}} \rightarrow \mathbb{G}_{\mathrm{m}}^{n}$ gives an isomorphism between $\mathbb{G}_{\mathrm{m}}$ and $T_{\boldsymbol{a}}$.

For subvarieties of tori, fibered products like those in (3.1) can be expressed in more concrete terms. The next lemma gives such an expression for the case of hypersurfaces.

Lemma 4.1. Let $F \in \mathbb{C}\left[\boldsymbol{x}^{ \pm 1}, z^{ \pm 1}\right], G \in \mathbb{C}\left[\boldsymbol{x}^{ \pm 1}\right] \backslash\{0\}$, and $A \in \mathbb{Z}^{n \times k}$. Let $W$ be the hypersurface of $\mathbb{G}_{\mathrm{m}}^{n+1} \backslash V(G)$ defined by $F, \pi: W \rightarrow \mathbb{G}_{\mathrm{m}}^{n}$ the map defined by $\pi(\boldsymbol{x}, z)=\boldsymbol{x}$, and $\lambda: \mathbb{G}_{\mathrm{m}}^{k} \rightarrow \mathbb{G}_{\mathrm{m}}^{n}$ the group morphism defined by $\lambda(\boldsymbol{t})=\boldsymbol{t}^{A}$. Then $\mathbb{G}_{\mathrm{m}}^{k} \times_{\lambda, \pi} W$ is isomorphic to the subscheme of $\mathbb{G}_{\mathrm{m}}^{k+1} \backslash V\left(G\left(\boldsymbol{t}^{A}\right)\right)$ defined by $F\left(\boldsymbol{t}^{A}, z\right)$.

Proof. The maps $\pi$ and $\lambda$ correspond to the morphisms of $\mathbb{C}$-algebras

$$
\mathbb{C}\left[\boldsymbol{x}^{ \pm 1}\right] \longrightarrow \mathbb{C}\left[\boldsymbol{x}^{ \pm 1}, z^{ \pm 1}\right]_{G} / F \quad \text { and } \quad \mathbb{C}\left[\boldsymbol{x}^{ \pm 1}\right] \longrightarrow \mathbb{C}\left[\boldsymbol{t}^{ \pm 1}\right] \simeq \mathbb{C}\left[\boldsymbol{x}^{ \pm 1}, \boldsymbol{t}^{ \pm 1}\right] /\left(\boldsymbol{x}-\boldsymbol{t}^{A}\right),
$$

and the fibered product $\mathbb{G}_{\mathrm{m}}^{k} \times_{\lambda, \pi} W$ is the scheme associated to the tensor product

$$
\mathbb{C}\left[\boldsymbol{x}^{ \pm 1}, z^{ \pm 1}\right]_{G} / F \otimes_{\mathbb{C}\left[\boldsymbol{x}^{ \pm 1}\right]} \mathbb{C}\left[\boldsymbol{x}^{ \pm 1}, \boldsymbol{t}^{ \pm 1}\right] /\left(\boldsymbol{x}-\boldsymbol{t}^{A}\right) .
$$

This tensor product is isomorphic to the $\mathbb{C}$-algebra

$$
\mathbb{C}\left[\boldsymbol{x}^{ \pm 1}, z^{ \pm 1}, \boldsymbol{t}^{ \pm 1}\right]_{G} /\left(F, \boldsymbol{x}-\boldsymbol{t}^{A}\right) \simeq \mathbb{C}\left[z^{ \pm 1}, \boldsymbol{t}^{ \pm 1}\right]_{G\left(\boldsymbol{t}^{A}\right)} /\left(F\left(\boldsymbol{t}^{A}, z\right)\right),
$$

which gives the statement.

Lemma 4.2. Let $f \in \mathbb{C}(t)[z]$ be an irreducible polynomial of degree $d \geq 1$, and such that $f\left(t^{m}, z\right)$ is reducible for some $m \in \mathbb{N}$. There is $e \in \mathbb{N}$ dividing $\operatorname{gcd}(m, d)$ such that $f\left(t^{e}, z\right)$ is also reducible.

Proof. The proof relies on the action of torsion points on irreducible factors as in Zan10, Proposition 2.1].

By Lemma 4.1, the subscheme of $\mathbb{G}_{\mathrm{m}}^{2}$ defined by $f\left(t^{m}, z\right)$ is isomorphic to the pullback $[m]^{*} V(f)$, with $[m]$ the $m$-th multiplication map of $\mathbb{G}_{\mathrm{m}}$. By Lemma 3.7, this pullback is reduced, and so $f\left(t^{m}, z\right)$ is separable. Consider its decomposition into distinct irreducible factors

$$
f\left(t^{m}, z\right)=\prod_{i=1}^{k} p_{i}
$$

with $k \geq 2$. 
The group $\mu_{m}$ of $m$-th roots of the unity acts on the set of these irreducible factors by $p_{i}(t, z) \mapsto p_{i}(\zeta \cdot t, z), i=1, \ldots, k$, for $\zeta \in \mu_{m}$. Let $\mathcal{P} \subset\left\{p_{1}, \ldots, p_{k}\right\}$ be a nonempty orbit of this action. The polynomial

$$
\prod_{p \in \mathcal{P}} p
$$

is invariant under the action of $\mu_{m}$, and so it is of the form $g\left(t^{m}, z\right)$ with $g \in \mathbb{C}(t)[z]$. This product is a nontrivial factor of $f\left(t^{m}, z\right)$, and so $g$ coincides with $f$ up to a scalar. It follows that $\mathcal{P}=\left\{p_{1}, \ldots, p_{k}\right\}$ and so the action is transitive. In particular, all the $p_{i}$ 's have the same degree in the variable $z$, and so this degree is positive and $k \mid d$.

The stabilizer of an irreducible factor $p_{i}$ is a subgroup of $\mu_{m}$, hence it is of the form $\mu_{l}$ with $l \mid m$. Since the action is transitive and $\mu_{m}$ is abelian, this subgroup does not depend on the choice of $p_{i}$. Moreover, $m / l$ is equal to $k$, the number of irreducible factors of $f\left(t^{m}, z\right)$, also because of the transitivity of the action.

By the invariance of each $p_{i}$ under the action of $\mu_{l}$, there is $q_{i} \in \mathbb{C}(t)[z] \backslash \mathbb{C}(t)$ with $p_{i}=q_{i}\left(t^{l}, z\right)$. It follows from 4.1 that

$$
f\left(t^{e}, z\right)=\prod_{i=1}^{k} q_{i}(t, z),
$$

with $e=m / l$. Clearly $e \mid m$ and as explained, $e=k$, and so this quantity also divides $d$, completing the proof.

Lemma 4.3. Let $F \in \mathbb{C}[\boldsymbol{x}, z]$ be an irreducible polynomial of degree $d \geq 1$ in the variable $z$, and $G \in \mathbb{C}[\boldsymbol{x}] \backslash\{0\}$ its leading coefficient.

(1) Let $W=V(F) \backslash V(G) \subset \mathbb{G}_{\mathrm{m}}^{n+1}$ and $\pi: W \rightarrow \mathbb{G}_{\mathrm{m}}^{n}$ the map defined by $\pi(\boldsymbol{x}, z)=$ $\boldsymbol{x}$. The image of $\pi$ is the open subset $\mathbb{G}_{\mathrm{m}}^{n} \backslash V(G)$ of $\mathbb{G}_{\mathrm{m}}^{n}$, and this map is finite onto this open subset.

(2) There is a finite subset $\Delta_{F}$ of $\mathbb{Z}^{n}$ such that for $\boldsymbol{a} \in \mathbb{Z}^{n}$ with $\langle\boldsymbol{c}, \boldsymbol{a}\rangle \neq 0$ for all $c \in \Delta_{F}$, the polynomial $F\left(t^{a}, z\right)$ has degree $d$ in the variable $z$.

(3) If $A \in \mathbb{Z}^{n \times n}$ is nonsingular, then $F\left(\boldsymbol{x}^{A}, z\right)$ has no nontrivial factors in $\mathbb{C}\left[\boldsymbol{x}^{ \pm 1}\right]_{G}$.

Proof. For the first statement, the image of the map $\pi$ is contained in the open set $U=\mathbb{G}_{\mathrm{m}}^{n} \backslash V(G)$. The induced map $W \rightarrow U$ corresponds to the morphism of $\mathbb{C}$-algebras

$$
\mathbb{C}\left[\boldsymbol{x}^{ \pm 1}\right]_{G} \longleftrightarrow \mathbb{C}\left[\boldsymbol{x}^{ \pm 1}, z\right]_{G} /(F) .
$$

This morphism is an integral extension because the leading term $G$ is invertible in $\mathbb{C}\left[\boldsymbol{x}^{ \pm 1}\right]_{G}$, and so the map $W \rightarrow U$ is finite and, a fortiori, surjective.

For the second statement, write $G=\sum_{j=1}^{r} G_{j} \boldsymbol{x}^{\boldsymbol{c}_{j}}$ with $G_{j} \in \mathbb{C}^{\times}$and $\boldsymbol{c}_{j} \in \mathbb{N}^{n}$, $j=1, \ldots, r$, and consider the finite subset of $\mathbb{Z}^{n}$ given by

$$
\Delta_{F}=\left\{\boldsymbol{c}_{j}-\boldsymbol{c}_{1} \mid j=2, \ldots, r\right\} .
$$

For $\boldsymbol{a} \in \mathbb{Z}^{n}$ with $\langle\boldsymbol{c}, \boldsymbol{a}\rangle \neq 0$ for all $\boldsymbol{c} \in \Delta_{F}$, we have that $G\left(t^{\boldsymbol{a}}\right) \neq 0$ and so $\operatorname{deg}_{z}\left(F\left(t^{a}, z\right)\right)=d$.

As for Lemma 4.2, the proof of the last assertion relies on the action of torsion points on irreducible factors, and so we only sketch it. Using Lemmas 4.1 and 3.7, we show that $F\left(\boldsymbol{x}^{A}, z\right)$ is separable. Let

$$
F\left(\boldsymbol{x}^{A}, z\right)=\prod_{i=1}^{k} P_{i}
$$


the decomposition of this Laurent polynomial into distinct irreducible factors. The action of the finite group $\left\{\boldsymbol{x} \in \mathbb{G}_{\mathrm{m}}^{n} \mid \boldsymbol{x}^{A}=1\right\}$ on the the sets of these irreducible factors is transitive, and so the $P_{i}$ 's have the same degree with respect to the variable $z$. Hence for $i=1, \ldots, k$, we have that $k \operatorname{deg}_{z}\left(P_{i}\right)=d \geq 1$. In particular, $\operatorname{deg}_{z}\left(P_{i}\right) \geq 1$, proving the statement.

Proof of Theorem 1.4. The statement of this result, restricted to primitive vectors $\boldsymbol{a} \in \mathbb{Z}^{n}$, is a specialization of Theorem 3.4. To see this, first reduce, multiplying by a suitable monomial, to the case when $F$ is an irreducible polynomial in $\mathbb{C}[\boldsymbol{x}, z]$ of degree $d \geq 1$ in the variable $z$. Set $W=V(F) \backslash V(G)$ and consider the map

$$
\pi: W \longrightarrow \mathbb{G}_{\mathrm{m}}^{n}
$$

defined by $\pi(\boldsymbol{x}, z)=\boldsymbol{x}$ for $(\boldsymbol{x}, z) \in W$. The quasi-projective variety $W$ is irreducible and, by Lemma 4.3 1, this map is dominant and finite onto its image, the open subset $U=\mathbb{G}_{\mathrm{m}}^{n} \backslash V(G)$ of $\mathbb{G}_{\mathrm{m}}^{n}$.

Let $\Lambda$ be a finite subset of isogenies of $\mathbb{G}_{\mathrm{m}}^{n}$ and $\mathcal{E}$ a finite union of proper subtori of $\mathbb{G}_{\mathrm{m}}^{n}$ satisfying the conclusion of Theorem 3.4 applied to this map. Set then $\Phi_{1}$ for the finite subset of nonsingular matrices in $\mathbb{Z}^{n \times n}$ corresponding to the isogenies in $\Lambda$, and $\Sigma_{1}$ for a finite subset of nonzero vectors of $\mathbb{Z}^{n}$ such that

$$
\mathcal{E} \subset \bigcup_{c \in \Sigma_{1}} V\left(\boldsymbol{x}^{c}-1\right)
$$

For a primitive vector $\boldsymbol{a} \in \mathbb{Z}^{n}$, set $T_{\boldsymbol{a}}$ for the 1-dimensional subtorus defined as the image of the group morphism $\mathbb{G}_{\mathrm{m}} \rightarrow \mathbb{G}_{\mathrm{m}}^{n}$. This map gives an isomorphism between $\mathbb{G}_{\mathrm{m}}$ and $T_{\boldsymbol{a}}$. By Lemma 4.1, the fiber $\pi^{-1}\left(T_{\boldsymbol{a}}\right)$ is isomorphic to the subscheme of $\mathbb{G}_{\mathrm{m}}^{2} \backslash V\left(G\left(t^{\boldsymbol{a}}\right)\right)$ defined by $F\left(t^{\boldsymbol{a}}, z\right)$. For the isogeny $\lambda$ associated to a nonsingular matrix $M \in \Phi_{1}$, the same result shows that $\lambda^{*} W$ is isomorphic to the subscheme of $\mathbb{G}_{\mathrm{m}}^{n+1} \backslash V(G)$ defined by $F\left(\boldsymbol{x}^{M}, z\right)$.

The three alternatives from Theorem 3.4 applied to the map $\pi$, the subtorus $T_{\boldsymbol{a}}$ and the point $p=(1, \ldots, 1) \in \mathbb{G}_{\mathrm{m}}^{n}(\mathbb{C})$, then boil down to those in the theorem under examination, as explained below.

(1) Suppose that $T_{\boldsymbol{a}} \subset \mathcal{E}$. By 4.2 , there is $\boldsymbol{c} \in \Sigma_{1}$ with $\langle\boldsymbol{c}, \boldsymbol{a}\rangle=0$.

(2) Else suppose that there is an isogeny $\lambda \in \Lambda$ with $\lambda^{*} W$ reducible and a subtorus $T^{\prime}$ of $\mathbb{G}_{\mathrm{m}}^{n}$ with $\lambda$ inducing an isomorphism between $T^{\prime}$ and $T_{\boldsymbol{a}}$. For $M \in \Phi_{1}$ the nonsingular matrix associated to $\lambda$, we have that $\boldsymbol{a} \in \operatorname{im}(B)$ and, by Lemma 4.1, $F\left(\boldsymbol{x}^{M}, z\right)$ is reducible.

(3) Else suppose that $\pi^{-1}\left(T_{\boldsymbol{a}}\right)$ is irreducible in $\mathbb{G}_{\mathrm{m}}^{2} \backslash V(G)$. By Lemma 4.1, this implies that $F\left(t^{a}, z\right)$ is irreducible in $\mathbb{C}(t)\left[z^{ \pm 1}\right]$.

We next enlarge these finite sets to cover the rest of the cases. Let $d \geq 1$ be the degree of $F$ in the variable $z$, and let $e$ be a divisor of $d$. If $F\left(\boldsymbol{x}^{e}, z\right)$ is irreducible, we respectively denote by $\Phi_{e}$ and $\Sigma_{e}$ the finite subsets of nonsingular matrices in $\mathbb{Z}^{n \times n}$ and of nonzero vectors of $\mathbb{Z}^{n}$ given by the application of Theorem 3.4 to this polynomial. Otherwise, we set $\Phi_{e}=\left\{I_{n}\right\}$ with $I_{n}$ the identity matrix of $\mathbb{Z}^{n \times n}$, and $\Sigma_{e}=\emptyset$. Set also $\Delta$ for the finite subset of nonzero vectors in $\mathbb{Z}^{n}$ associated to $F$ by Lemma 4.3 2). Set then

$$
\Phi=\bigcup_{e \mid d} e \Phi_{e} \quad \text { and } \quad \Sigma=\Delta \cup \bigcup_{e \mid d} \Sigma_{e} .
$$

By Theorem 3.4 and the previous analysis, the statement holds for all vectors of the form $e \boldsymbol{b}$ with $\boldsymbol{b} \in \mathbb{Z}^{n}$ primitive and $e \mid d$. 
Given an arbitrary vector $\boldsymbol{a} \in \mathbb{Z}^{n}$, write $\boldsymbol{a}=m \boldsymbol{b}$ with $m \in \mathbb{N}$ and $\boldsymbol{b} \in \mathbb{Z}^{n}$ primitive, and set

$$
f=F\left(t^{b}, z\right) \in \mathbb{C}\left[t^{ \pm 1}, z\right] .
$$

Suppose that neither (1) nor (2) hold for $\boldsymbol{a}$. Let $e \in \mathbb{N}$ be a common divisor of $d$ and $m$. A fortiori, these conditions do neither hold for $e \boldsymbol{b}$ and, as explained before,

$$
f\left(t^{e}, z\right)=F\left(t^{e b}, z\right)
$$

is irreducible in $\mathbb{C}(t)[z]$. By Lemma 4.2, we have that $F_{\boldsymbol{a}}=f\left(t^{m}, z\right)$ is irreducible in $\mathbb{C}(t)[z]$, giving the condition (3) for $\boldsymbol{a}$ and concluding the proof.

Remark 4.4. Using the toric Bertini's theorem 3.4 for cosets of arbitrary dimension, the present polynomial version in Theorem 1.4 might be extended to $k$-parameter monomial maps for any $k$, and also to arbitrary translates of these monomial maps.

We have kept the present more restricted statement for the sake of simplicity, and also because it is sufficient for our application.

\section{Factorization of Sparse polynomials}

Here we prove the results on the factorization of Laurent polynomials announced in the introduction and in Section 2. Theorem 2.4 is easily seen to be implied by the following statement.

Theorem 5.1. Let $F \in \mathbb{C}\left[\boldsymbol{x}^{ \pm 1}, z^{ \pm 1}\right]$ without nontrivial factors in $\mathbb{C}\left[\boldsymbol{x}^{ \pm 1}\right]$. There are finite sets $\Omega^{0} \subset \mathbb{Z}^{n \times n}$ of nonsingular matrices and $\Gamma \subset \mathbb{Z}^{n}$ of nonzero vectors satisfying the property that, for $\boldsymbol{a} \in \mathbb{Z}^{n} \backslash\{\mathbf{0}\}$, one of the next alternatives holds:

(1) there is $\boldsymbol{c} \in \Gamma$ with $\langle\boldsymbol{c}, \boldsymbol{a}\rangle=0$;

(2) there are $M \in \Omega^{0}$ and $\boldsymbol{b} \in \mathbb{Z}^{n}$ with $\boldsymbol{a}=M \boldsymbol{b}$ such that if

$$
F\left(\boldsymbol{x}^{M}, z\right)=\prod_{P} P^{e_{P}}
$$

is the irreducible factorization of $F\left(\boldsymbol{x}^{M}, z\right)$ in $\mathbb{C}\left[\boldsymbol{x}^{ \pm 1}, z^{ \pm 1}\right]$, then

$$
F\left(t^{\boldsymbol{a}}, z\right)=\prod_{P} P\left(t^{\boldsymbol{b}}, z\right)^{e_{P}}
$$

is the irreducible factorization of $F\left(t^{a}, z\right)$ in $\mathbb{C}(t)\left[z^{ \pm 1}\right]$.

Proof. We proceed by induction on $\operatorname{deg}_{z}(F)$. When $\operatorname{deg}_{z}(F)=0$, the statement is trivial, and so we assume that $\operatorname{deg}_{z}(F) \geq 1$.

If $F$ is irreducible, we respectively denote by $\Phi$ and $\Sigma$ the finite sets of nonsingular matrices in $\mathbb{Z}^{n \times n}$ and of nonzero vectors in $\mathbb{Z}^{n}$ from Theorem 5.1 applied to this Laurent polynomial. If $F$ is reducible, we set $\Phi=\left\{I_{n}\right\}$ and $\Sigma=\emptyset$.

Let $\boldsymbol{a} \in \mathbb{Z}^{n}$. When $F$ is irreducible, if the condition (1) in Theorem 1.4 holds, then the condition (1) in Theorem 5.1 also holds by taking $\Gamma$ as any finite set containing $\Sigma$. Still in the irreducible case, if the condition (3) in Theorem 1.4 holds, the Laurent polynomial $F\left(t^{a}, z\right)$ is irreducible, and the condition (1) in Theorem 5.1 holds provided that $\Omega^{0}$ contains $I_{n}$.

Else, suppose that the condition (2) in Theorem 1.4 holds, that is, there are $M \in \Phi$ and $\boldsymbol{b} \in \mathbb{Z}^{n}$ with $\boldsymbol{a}=M \boldsymbol{b}$ and $F\left(\boldsymbol{x}^{M}, z\right)$ is reducible. Let

$$
F\left(\boldsymbol{x}^{M}, z\right)=F_{1} F_{2}
$$

be a nontrivial factorization. By Lemma $4.3(3), F\left(\boldsymbol{x}^{M}, z\right)$ has no factors in $\mathbb{C}\left[\boldsymbol{x}^{ \pm 1}\right]$. Hence $\operatorname{deg}_{z}\left(F_{i}\right)<\operatorname{deg}_{z}(F), i=1,2$, and by induction, Theorem 5.1 holds for these 
Laurent polynomials. Let $\Omega_{i}^{0}$ and $\Gamma_{i}$ respectively denote the finite sets of nonsingular matrices in $\mathbb{Z}^{n \times n}$ and of nonzero vectors in $\mathbb{Z}^{n}$ whose existence is assured by this theorem.

By construction, either there is a vector $\boldsymbol{c} \in \Gamma_{1} \cup \Gamma_{2}$ with $\langle\boldsymbol{c}, \boldsymbol{b}\rangle=0$, or we can find $M_{i} \in \Omega_{i}$ and $\boldsymbol{b}_{i} \in \mathbb{Z}^{n}$ with $\boldsymbol{b}=M_{i} \boldsymbol{b}_{i}$ and a decomposition

$$
F_{i}\left(\boldsymbol{x}^{M_{i}}, z\right)=\prod_{j=1}^{k_{i}} F_{i, j}
$$

with $F_{i, j}\left(t^{\boldsymbol{b}_{i}}, z\right)$ irreducible in $\mathbb{C}(t)\left[s^{ \pm 1}\right]$ for all $i, j$.

Set

$$
\Gamma=\left\{\operatorname{adj}(M) \boldsymbol{c} \mid M \in \Phi, \boldsymbol{c} \in \Gamma_{1} \cup \Gamma_{2}\right\}
$$

with $\operatorname{adj}(M)$ the adjoint matrix of $M$. If $\left\langle\boldsymbol{c}^{\prime}, \boldsymbol{a}\right\rangle \neq 0$ for all $\boldsymbol{c}^{\prime} \in \Gamma$, then $\langle\boldsymbol{c}, \boldsymbol{b}\rangle \neq 0$ for all $\boldsymbol{c} \in \Gamma_{1} \cup \Gamma_{2}$ and so $F_{i, j}\left(t^{b_{i}}, z\right)$ is irreducible in $\mathbb{C}(t)\left[s^{ \pm 1}\right]$ for all $i, j$.

Consider the lattices $K_{i}=\operatorname{im}\left(M_{i}\right), i=1,2$, and set $K=K_{1} \cap K_{2}$. Since $K$ is also a lattice, there is a nonsingular matrix $M^{\prime} \in \mathbb{Z}^{n \times n}$ with $K=\operatorname{im}\left(M^{\prime}\right)$ and, since $K \subseteq K_{i}$, there are nonsingular matrices $N_{i}, i=1,2$, with $M^{\prime}=M_{i} N_{i}$. Furthermore, $\boldsymbol{b} \in K$ implies that there is $\boldsymbol{b}^{\prime} \in \mathbb{Z}^{n}$ with $\boldsymbol{b}=M^{\prime} \boldsymbol{b}^{\prime}=M_{i} N_{i} \boldsymbol{b}^{\prime}$. Hence $\boldsymbol{b}_{i}=M_{i}^{-1} \boldsymbol{b}=N_{i} \boldsymbol{b}^{\prime}$ and

$$
F\left(\boldsymbol{x}^{M M^{\prime}}, z\right)=F_{1}\left(\boldsymbol{x}^{M_{1} N_{1}}, z\right) F_{2}\left(\boldsymbol{x}^{M_{2} N_{2}}, z\right)=\prod_{i=1}^{2} \prod_{j=1}^{r_{i}} F_{i, j}\left(\boldsymbol{x}^{N_{i}}, z\right) .
$$

Set $M^{\prime \prime}=M M^{\prime}, G_{i, j}=F_{i, j}\left(\boldsymbol{x}^{B_{i}}, z\right)$ and consider the decomposition

$$
F\left(\boldsymbol{x}^{M^{\prime \prime}}, z\right)=\prod_{i=1}^{2} \prod_{j=1}^{r_{i}} G_{i, j}
$$

We have $\boldsymbol{a}=M \boldsymbol{b}=M^{\prime \prime} \boldsymbol{b}^{\prime}$ and

$$
G_{i, j}\left(t^{\boldsymbol{b}^{\prime}}, z\right)=F_{i, j}\left(t^{B_{i} \boldsymbol{b}^{\prime}}, z\right)=F_{i, j}\left(t^{\boldsymbol{b}_{i}}, z\right)
$$

is irreducible in $\mathbb{C}(t)\left[s^{ \pm 1}\right]$ for all $i, j$. The statement follows by taking $\Omega^{0}$ as any finite set containing all the matrices of the form $M M^{\prime}$ for $M \in \Phi$, and $\Gamma$ as in (5.1).

We conclude by giving the proof of our main result.

Proof of Theorem 1.3. We proceed by induction on $n$. When $n=0$ the statement is trivial, and so we assume that $n \geq 1$. Let $F \in \mathbb{C}\left[\boldsymbol{x}^{ \pm 1}, z^{ \pm 1}\right]$ and write

$$
F=C F^{\prime}
$$

with $C \in \mathbb{C}\left[\boldsymbol{x}^{ \pm 1}\right]$ and $F^{\prime} \in \mathbb{C}\left[\boldsymbol{x}^{ \pm 1}, z^{ \pm 1}\right]$ without nontrivial factors in $\mathbb{C}\left[\boldsymbol{x}^{ \pm 1}\right]$. By Lemma 4.3 2), there is a finite subset $\Delta \subset \mathbb{Z}^{n}$ such that $C\left(t^{b}\right) \neq 0$ for all $\boldsymbol{b} \in \mathbb{Z}^{n}$ with $\langle\boldsymbol{c}, \boldsymbol{b}\rangle \neq 0$ for all $\boldsymbol{c} \in \Delta$. Let also $\Omega^{0} \subset \mathbb{Z}^{n \times n}$ and $\Gamma \in \mathbb{Z}^{n}$ be the finite subsets given by Theorem 5.1 applied to $F^{\prime}$.

Let $\boldsymbol{a} \in \mathbb{Z}^{n}$. When $\langle\boldsymbol{c}, \boldsymbol{a}\rangle \neq 0$ for all $\boldsymbol{c} \in \Gamma \cup \Delta$, Theorem 5.1 2 implies the statement, provided that we choose any finite subset $\Omega \subset \mathbb{Z}^{n \times n}$ containing $\Omega^{0}$.

Otherwise, suppose that there is $\boldsymbol{c} \in \Gamma \cup \Delta$ with $\langle\boldsymbol{c}, \boldsymbol{a}\rangle=0$. If $C\left(t^{\boldsymbol{a}}, z\right)=0$, we add to the finite set $\Omega$ the matrix $M \in \mathbb{Z}^{n \times n}$ made by adding to $n-1$ zero columns to the vector $\boldsymbol{a}$. Otherwise, choose a matrix $L \in \mathbb{Z}^{n \times(n-1)}$ defining a linear map $\mathbb{Z}^{n-1} \rightarrow \mathbb{Z}^{n}$ whose image is the submodule $c^{\perp} \cap \mathbb{Z}^{n}$, and a vector $\boldsymbol{d} \in \mathbb{Z}^{n-1}$ with $\boldsymbol{a}=L \boldsymbol{d}$. Let $\boldsymbol{u}=\left(u_{1}, \ldots, u_{n-1}\right)$ be a set of $n-1$ variables and set

$$
G=F^{\prime}\left(\boldsymbol{u}^{L}\right) \in \mathbb{C}\left[\boldsymbol{u}^{ \pm 1}, z^{ \pm 1}\right]
$$


By the inductive hypothesis, there is a finite subset $\Omega_{c} \subset \mathbb{Z}^{(n-1) \times(n-1)}$ satisfying the statement of Theorem 1.3 applied to this Laurent polynomial. In particular, there are $N \in \Omega_{\boldsymbol{c}}$ and $\boldsymbol{e} \in \mathbb{Z}^{n-1}$ with $\boldsymbol{d}=N \boldsymbol{e}$ such that, for an irreducible factor $Q$ of $G\left(\boldsymbol{u}^{N}, z\right)$, we have that $Q\left(t^{e}, z\right)$ is, as a Laurent polynomial in $\mathbb{C}(t)\left[z^{ \pm 1}\right]$, either a unit or an irreducible factor of $G\left(t^{d}, z\right)$.

We have that $G\left(\boldsymbol{u}^{N}, z\right)=F^{\prime}\left(\boldsymbol{u}^{L N}, z\right)$, and so $Q$ is an irreducible factor of this latter Laurent polynomial. Moreover, $\boldsymbol{a}=L N \boldsymbol{e}$. Enlarging the matrix $L N \in \mathbb{Z}^{n \times(n-1)}$ to a matrix $M \in \mathbb{Z}^{n \times n}$ by adding to it a zero column at the end, and similarly enlarging the vector $\boldsymbol{e}$ to a vector $\boldsymbol{b} \in \mathbb{Z}^{n}$ by adding to it a zero entry at the end, the previous equalities are preserved with $M$ and $\boldsymbol{b}$ in the place of $L N$ and $\boldsymbol{e}$. Hence, $\boldsymbol{a}=M \boldsymbol{b}$ and, if $Q\left(x_{1}, \ldots, x_{n-1}\right)$ is an irreducible factor of $F^{\prime}\left(\boldsymbol{x}^{M}, z\right)$, then $Q\left(t^{e}, z\right)=Q\left(t^{\boldsymbol{b}}, z\right)$ is, as a Laurent polynomial in $\mathbb{C}(t)\left[z^{ \pm 1}\right]$, either a unit or an irreducible factor of $G\left(t^{d}, z\right)=F\left(t^{a}, z\right)$.

The statement then follows by also also adding to $\Omega$ all the matrices $M \in \mathbb{Z}^{n \times n}$ constructed in this way.

Remark 5.2. In the setting of Theorem 1.3 , the bivariate Laurent polynomials $F_{\boldsymbol{a}}$ can be defined as the pullback of the multivariate Laurent polynomial $F$ by the 2-parameter monomial map $(t, z) \mapsto(t, z)^{A}$ given by the matrix

$$
A=\left(\begin{array}{cc}
0 & 1 \\
a_{1} & 0 \\
\vdots & \vdots \\
a_{n} & 0
\end{array}\right) \in \mathbb{Z}^{(n+1) \times 2}
$$

In Conjecture 1.5 applied to $F$ and $k=2$, one can consider all matrices in $\mathbb{Z}^{(n+1) \times 2}$, and so its setting is more general than that of Theorem 1.3 .

On the other hand, the conclusion of Conjecture 1.5 in this situation is slightly weaker than that of Theorem 1.3 , since it does not give the irreducible factorization of the $F_{\boldsymbol{a}}$ in $\mathbb{C}(t)\left[z^{ \pm 1}\right]$, but rather its irreducible factorization modulo the Laurent polynomials of the form $f\left(t^{d_{1}} z^{d_{2}}\right)$ for a univariate $f$ and $d_{1}, d_{2} \in \mathbb{Z}$.

\section{REFERENCES}

[AKS07] M. Avendaño, T. Krick, and M. Sombra, Factoring bivariate sparse (lacunary) polynomials, J. Complexity 23 (2007), 193-216.

[ASZ17] F. Amoroso, M. Sombra, and U. Zannier, Unlikely intersections and multiple roots of sparse polynomials, Math. Z. 287 (2017), 1065-1081.

[FGS08] M. Filaseta, A. Granville, and A. Schinzel, Irreducibility and greatest common divisor algorithms for sparse polynomials, Number theory and polynomials, London Math. Soc. Lecture Notes Ser., vol. 352, Cambridge Univ. Press, 2008, pp. 155-176.

[FMZ18] C. Fuchs, V. Mantova, and U. Zannier, On fewnomials, integral points, and a toric version of Bertini's theorem, J. Amer. Math. Soc. 31 (2018), 107-134.

[Gre16] B. Grenet, Bounded-degree factors of lacunary multivariate polynomials, J. Symbolic Comput. 75 (2016), 171-192.

[Har77] R. Hartshorne, Algebraic geometry, Graduate Texts in Math., vol. 52, Springer-Verlag, 1977.

[Jou83] J.-P. Jouanolou, Théorèmes de Bertini et applications, Progr. Math., vol. 42, Birkhäuser, 1983.

[KK06] E. Kaltofen and P. Koiran, Finding small degree factors of multivariate supersparse (lacunary) polynomials over algebraic number fields, ISSAC 2006, ACM, 2006, pp. 162-168.

[Len99] H. W. Lenstra, Jr., On the factorization of lacunary polynomials, Number theory in progress, vol. 1 (Zakopane-Kościelisko, 1997), de Gruyter, 1999, pp. 277-291.

[Mum88] D. Mumford, The red book of varieties and schemes, Lecture Notes in Math., vol. 1358, Springer-Verlag, 1988. 
[Sch65] A. Schinzel, On the reducibility of polynomials and in particular of trinomials, Acta Arith. 11 (1965), 1-34.

[Sch70] _ Reducibility of lacunary polynomials. I, Acta Arith. 16 (1969/1970), 123-159.

[Sch90] _ An analog of Hilbert's irreducibility theorem, Number theory (Banff, AB, 1988), de Gruyter, Berlin, 1990, pp. 509-514. MR 1106684

[Sch00] - Polynomials with special regard to reducibility. With an appendix by Umberto Zannier, Encyclopedia Math. Appl., vol. 77, Cambridge Univ. Press, 2000.

[Zan10] U. Zannier, Hilbert irreducibility above algebraic groups, Duke Math. J. 153 (2010), 397-425.

[ZS75] O. Zariski and P. Samuel, Commutative algebra. Vol. II, Graduate Texts in Math., vol. 29, Springer-Verlag, 1975.

Laboratoire de mathématiques Nicolas Oresme, CNRS UMR 6139, Université de Caen. BP 5186, 14032 Caen Cedex, France

E-mail address: francesco.amoroso@unicaen.fr

URL: http://www .math.unicaen.fr/ amoroso/

Institució Catalana de Recerca i Estudis Avançats (ICREA). Passeig Lluís CompaNys 23, 08010 BARCELONA, SPAin

Departament de Matemàtiques i Informàtica, Universitat de Barcelona (UB). Gran Via 585, 08007 Barcelona, Spain

E-mail address: sombra@ub.edu

$U R L:$ http://www . maia.ub.edu/ sombra 